\title{
Gli effetti della live sax music nei pazienti sottoposti a emodialisi: uno studio randomizzato controllato
}

\section{Francesco Burrai}

Professore a contratto di Terapia Complementare, Università degli Studi di Bologna e Coordinatore Didattico, Corso di Laurea in Infermieristica, BO1-AOSP S. Orsola Malpighi, Bologna; Direttore Scientifico dell'Italian EDTNA/ERCA Research Center

\begin{abstract}
Effects of live sax music in patients undergoing haemodialysis: a randomized controlled trial
Background: A few studies have focused on the effect of music in patients undergoing haemodialysis.

Objectives: The determination of the effects of live saxophone music on various physiological parameters and pain, mood and itching levels.

Design: A randomised controlled study with 114 patients undergoing haemodialysis with a control group and an experimental group who received 30-minutes of live saxophone music therapy.

Measurements: Systolic and diastolic blood pressure, pulse rate, glycaemia, oxygen saturation, pain, mood and itching levels were measured before and after the live music performance, resulting in baseline and post-test values for the patients in the experimental group.

Results: The experimental group showed a statistically significant reduction in pain level, and an improvement in mood and itching levels and in the oxygen saturation.

Conclusions: Live saxophone music could be introduced to improve clinical and quality-of-life measures in patients undergoing haemodialysis.
\end{abstract}

Keywords: Music therapy, Holistic care, Holistic nursing, Complementary medicine

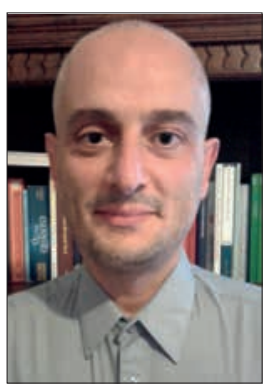

Francesco Burrai

\section{Introduzione}

Da secoli l'uomo riconosce l'efficacia terapeutica e psicosomatica della musica. La musica rappresenta una terapia complementare, non farmacologica, che riveste un ruolo importante nell'assistenza sanitaria (1). Nell'ultimo ventennio abbiamo assistito a un notevole incremento dell'impiego della musica in ambito infermieristico (2). L'utilizzo della musica è all'interno della Classificazione degli Interventi Infermieristici (NIC) (3) e il Joanna Briggs Institute ha assegnato il massimo punteggio a questo

Accepted: August 28, 2015

Published online: September 16, 2015

Indirizzo per la corrispondenza:

Prof. Francesco Burrai

Professore a contratto

Università di Bologna

Viale Ercolani 6

40138 Bologna

francesco.burrai2@unibo.it tipo di intervento olistico considerandolo un importante intervento per l'infermieristica (4). La musica ha assunto per la prima volta un ruolo terapeutico nel ridurre l'ansia e il dolore (5) e nel migliorare l'umore (6) e la qualità della vita (7) sia in pazienti che soffrivano di patologie croniche che in pazienti in stadio avanzato (8). Nell'assistenza infermieristica in emodialisi, la musica è utilizzata per migliorare lo stato emotivo del paziente (9), per ridurre le oscillazioni dei valori pressori (10), per ridurre l'ansia e migliorare la depressione (11), per migliorare il benessere (12), per ridurre la tensione psicofisica (13) e per garantire la durata della terapia dialitica (14). A oggi, non esistono RCT che abbiano studiato gli effetti della musica live con il sassofono sui parametri fisiologici, sul dolore, sull'umore e sul grado di prurito nei pazienti sottoposti a emodialisi.

\section{Framework concettuale}

Il modello concettuale, il framework utilizzato per questo studio, è quello psiconeuroendocrinoimmunologico. In questo modello, la musica, variabile indipendente del modello, può attivare le strutture cerebrali e, di conseguenza, tutti gli altri sistemi. I vari sistemi mostrano connessioni che coinvolgono direttamente il sistema nervoso autonomo, il sistema endocrino e il sistema immunitario. Pertanto, potenzialmente, la musica può modulare questi sistemi e le loro connessioni. II model- 
lo prende in considerazione sia gli aspetti psicologici sia quelli fisiologici in un'unica visione olistica, indicando l'azione della musica sui pazienti come segue:

1) la percezione del suono da parte del sistema uditivo e degli elementi di ritmo, tonalità e timbro sono ricodificati come segnali neuronali; 2) i segnali neuronali dal sistema nervoso centrale sono inviati al sistema nervoso autonomo, al sistema endocrino e al sistema immunitario in modo bidirezionale; 3) il sistema uditivo, il sistema nervoso centrale, il sistema nervoso autonomo, il sistema endocrino e il sistema immunitario possono influenzare direttamente lo stato fisiologico e la condizione psicologica del paziente; 4) il nuovo stato fisiologico e psicologico prodotto dall'azione della musica può influenzare i diversi outcome.

\section{Scopo}

Lo scopo di questo studio RCT è quello di testare un intervento non farmacologico in riferimento a diversi parametri fisiologici, il livello del dolore, lo stato dell'umore e l'intensità del prurito nei pazienti sottoposti a emodialisi.

\section{Quesito di ricerca}

Qual è l'efficacia dell'ascolto della musica rispetto al non ascolto della musica in relazione al miglioramento dell'umore, del dolore, del prurito, della pressione sanguigna sistolica e diastolica, della frequenza cardiaca, della glicemia e della la saturazione dell'ossigeno, nei pazienti sottoposti a emodialisi?

\section{Ipotesi di ricerca}

Queste le ipotesi generate dal framework concettuale:

1. I'umore sarà più elevato nel gruppo di intervento con la musica rispetto al gruppo controllo;

2. l'intensità del prurito e il livello del dolore saranno minori nel gruppo di intervento con la musica rispetto al gruppo controllo;

3. la pressione sanguigna sistolica e diastolica, la frequenza cardiaca e la glicemia saranno minori nel gruppo di intervento con la musica rispetto al gruppo controllo;

4. la saturazione dell'ossigeno sarà maggiore nel gruppo di intervento con la musica rispetto al gruppo controllo.

\section{Metodi}

\section{Disegno del trial}

Verrà utilizzato un disegno di tipo sperimentale pre-test post-test, monocentrico, non stratificato, con randomizzazione bilanciata (1:1), controllato verso non intervento e a due gruppi paralleli, condotto in Italia. II gruppo sperimentale ascolterà musica live con sax per un mese in aggiunta all'assistenza standard. II gruppo controllo avrà solo l'assistenza standard.

\section{Partecipanti}

Questo studio è in linea con la Dichiarazione di Helsinki.

I criteri di inclusione sono: 1) una diagnosi confermata di IRC; 2) trattamento emodialitico; 3) consenso informato firmato.

Saranno esclusi i pazienti se hanno: 1) acusia; 2) severi disordini neurologici; 3) severi disordini psichiatrici; 4) deficit cognitivi; 5) riduzione del livello di coscienza.

\section{Randomizzazione}

I partecipanti sono assegnati mediante randomizzazione semplice (numeri random generati da software) a ciascuno dei due gruppi. La sequenza di randomizzazione sarà generata utilizzando il software statistico on-line (www.randomization.org) con un rapporto di allocazione 1:1.

\section{Setting}

L'arruolamento dei pazienti è stato effettuato presso l'unità operativa di Emodialisi Stefoni dell'Azienda OspedalieroUniversitaria S. Orsola Malpighi di Bologna.

\section{Materiali}

Le musiche sono state eseguite con un Sax Contralto e un Sax Soprano.

\section{Intervento}

\section{Gruppo sperimentale}

In aggiunta all'assistenza standard, il gruppo sperimentale riceverà un intervento musicale attraverso l'ascolto di musica live eseguita con un Sax Contralto e un Sax Soprano.

La musica è stata pre-selezionata dal primo ricercatore in collaborazione con la sassofonista.

I pazienti appartenenti al gruppo sperimentale avevano la possibilità di scegliere, da una lista di 100 brani, 5 o 6 brani musicali di vario genere (inclusi brani rilassanti, allegri e vivaci), nonché il genere musicale preferito (tra cui pop, jazz, colonne sonore, musica classica e folcloristica). La durata totale dell'intervento era di circa 30 minuti per paziente. L'intervento era stato eseguito da un'infermiera, con una formazione di tipo olistico e un percorso musicale universitario. Lo strumento suonato era il sassofono, sia Contralto che Soprano. Lo strumento veniva suonato davanti al paziente durante il trattamento emodialitico, come mostrato nella Figura 1. 

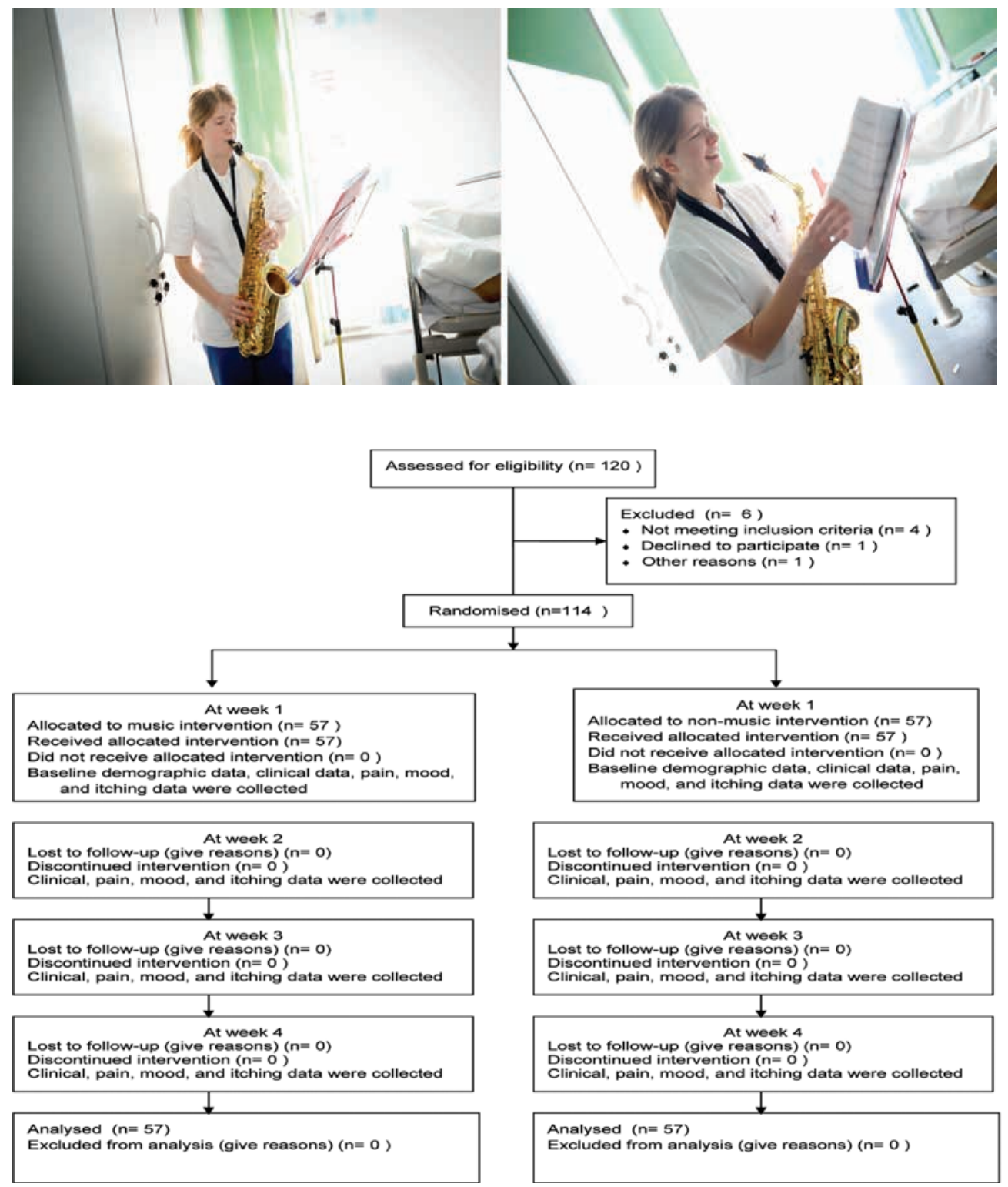

Fig. 1 - L'intervento musicale.

Fig. 2 - Flow Chart RCT.

\section{Gruppo controllo}

I pazienti del gruppo controllo riceveranno solo l'assistenza standard.

\section{Strumenti di misurazione}

Lo strumento di ricerca prevedeva 3 sezioni:

parte 1: raccolta dei dati demografici: età, sesso, stato civile, livello di istruzione, religione, ascolto della musica; parte 2: parametri fisiologici: pressione sanguigna sistolica e diastolica, frequenza cardiaca, glicemia e saturazione dell'ossigeno; parte 3: grado di intensità del dolore, rilevato con la Scala Visiva Analogica del Dolore (VASP), che misura l'intensità del dolore su una scala da 0 a 10, dove 0 rappresenta I' "assenza di dolore" e 10 il "peggior dolore percepito"; livello dell'umore, rilevato con la Scala Visiva Analogica dell'Umore (VASM), che misura il grado di umore da 0 a 10, dove 0 rappresenta "lo stato d'animo ottimale" e 10 "il peggior stato d'animo possibile"; livello di prurito, rilevato con la Scala Visiva Analogica per il Prurito (VASI), che misura l'intensità del prurito su una scala da 0 a 10, dove 0 rappresenta "assenza di prurito" e 10 "la maggiore intensità di prurito possibile". Le scale VAS forniscono misure valide, attendibili e concrete negli outcome considerati (15-17). La baseline e i post-test erano formati dalla raccolta dati delle 3 parti dello strumento di raccolta dati. Nel gruppo sperimentale, i dati dei post-test venivano raccolti immediatamente dopo i 30 minuti dell'intervento, mentre, nel gruppo controllo, i post-test venivano raccolti dopo 30 minuti di riposo (18). Lo studio è durato 4 settimane. 


\section{Sample Size}

Il sample size si basa sull'endpoint primario della qualità dell'umore misurato con la VASM. Considerando due gruppi bilanciati $(n 1=n 2)$ e un effect size medio $(d=0.5)$, con il livello di significatività $=0.05$ e l' $80 \%$ di potenza nel rilevare differenze tra i gruppi, sarebbero necessari un totale di 130 (n1 = $\mathrm{n} 2=60$ ) pazienti arruolati. Un effect size medio $=0.5$ implica una differenza campionaria attesa di 5 punti sulla scala VASM. Vista la natura ordinale della variabile e presupponendo una distribuzione normale dei punteggi, per mantenere la potenza al valore atteso (80\%), occorre moltiplicare per $\pi / 3$ (valore dell'efficienza asintotica relativa). Presupponendo, infine, un drop-out del 5\% per gruppo, sarà necessario arruolare 112 pazienti, 56 soggetti per gruppo.

\section{Outcome}

L'outcome primario è il livello di dolore, misurato con la scala VASP.

Gli outcome secondari sono: la condizione dell'umore, misurata con la scala VASM, il grado di intensità del prurito, misurato con la scala VASI, la pressione sanguigna sistolica e quella diastolica, misurate con sistema digitale via monitor, la frequenza cardiaca con sistema digitale via monitor, la glicemia, misurata con prelievo capillare, e la saturazione dell'ossigeno, misurata con saturimetro.

\section{Analisi statistica}

Per la descrizione dei dati clinici e sociodemografici abbiamo utilizzato una statistica descrittiva, usando le medie per gli indici di tendenza centrali, la deviazione standard per gli indici di dispersione e frequenza e percentuali per gli indici di distribuzione. Test T, Test $\chi 2$ e Fisher Test, sono stati usati per lo studio dell'omogeneità dei gruppi. Shapiro-Wilk Test è stato usato per la verifica della normalità. I risultati dei test hanno confermato l'appropriatezza dei test non parametrici. Nell'ambito del disegno di ricerca pre- e post-test, è stato calcolato il parametro Delta per misurare i cambiamenti tra le variabili dipendenti sia nel gruppo controllo che in quello sperimentale. Mann-Whitney $U$ Test è stato usato per studiare eventuali differenze statisticamente significative tra i gruppi. Wilcoxon Test è stato usato per studiare eventuali differenze statisticamente nei gruppi. $\alpha$ $=0.05$, usando un test a doppia coda. L'analisi statistica è stata eseguita usando SPSS ${ }^{\circledR} 18.0$ per Windows ${ }^{\circledR}$.

\section{Risultati \\ Campione}

I pazienti eleggibili erano 120; questi pazienti accedevano tramite programmazione all'unità operativa di dialisi dell'AOSP S. Orsola Malpighi, Bologna. Quattro pazienti furono esclu- si perché non rientravano nei criteri di inclusione e un paziente aveva rinunciato. Centoquattordici pazienti sono stati allocati in maniera randomizzata nel gruppo sperimentale ( $\mathrm{n}$ $=57$ ) e nel gruppo controllo $(n=57)$. II relativo diagramma di flusso viene mostrato nella Figura 2.

\section{Dati demografici}

L'età media dei partecipanti era di 68.2 (DS = 11.7); il 57.7\% era di sesso femminile $(n=65)$ e il $71.9 \%(n=82)$ era coniugato. La maggior parte dei pazienti possedeva un livello di istruzione scolastica superiore ( $n=48,42.1 \%$ ); la maggioranza era di religione cattolica ( $n=91,79.8 \%$ ). Tra i partecipanti, I' $86 \%$ $(n=98)$ ascoltava regolarmente la musica. Rispetto alle variabili demografiche, non sussistevano differenze statisticamente significative tra i due gruppi.

\section{Parametri fisiologici}

Come mostrato nella Tabella I, non furono trovate differenze statisticamente significative tra i due gruppi o al loro interno, a parte la saturazione dell'ossigeno, per la quale si sono registrate differenze statisticamente significative tra i due gruppi nel post-test $(p=0.000)$ e all'interno del gruppo controllo $(p=0.000)$.

\section{Dolore, umore e livelli di prurito}

Nel gruppo sperimentale sono state riscontrate differenze statisticamente significative connesse a dolore, umore e grado di prurito percepito $(p<0.05)$ e anche tra i due gruppi nel post-test $(p<0.05)$, come mostrato nella Tabella I.

I livelli del prurito hanno mostrato una riduzione statisticamente significativa del $75.7 \%(p=0.000)$; inoltre, si sono osservati un significativo incremento del $70 \%$ dell'umore e una riduzione significativa del dolore del 48.1\% ( $p=0.000)$, come mostrato nella Figura 3.

\section{Discussione}

In letteratura non esistono fonti in riferimento all'utilizzo della musica suonata con il sassofono ai pazienti sottoposti a emodialisi. I nostri risultati sono stati confrontati con una revisione sistematica Cochrane, la quale ha esaminato 30 $\mathrm{RCT}$, per un totale di 1.891 pazienti, e che riportava dati sugli effetti della musica in diverse tipologie di pazienti e sulle variabili come la saturazione dell'ossigeno, il dolore e i livelli dell'umore (19).

Questo nostro trial mostra un incremento statisticamente significativo della saturazione dell'ossigeno. La revisione sistematica della Cochrane (19), suggeriva che non si possono trarre conclusioni definitive riguardo all'effetto della musica sui livelli di saturazione dell'ossigeno negli adulti. Malgrado ciò, sono stati osservati importanti miglioramen- 
TABELLA I - Comparazione degli Outcome tra i gruppi

\begin{tabular}{|c|c|c|c|c|c|c|}
\hline \multirow[t]{2}{*}{ Outcome } & \multicolumn{4}{|c|}{ Gruppi } & \multicolumn{2}{|c|}{ Mann-Whitney } \\
\hline & \multicolumn{2}{|c|}{ Controllo $(n=57)$} & \multicolumn{2}{|c|}{ Sperimentale $(n=57)$} & U-Test & $p$ \\
\hline \multicolumn{7}{|c|}{ Pressione arteriosa sistolica } \\
\hline Baseline & $135.1(27.8)$ & $132.0[70.0-194.0]$ & $134.46(26.2)$ & $135,00[71.0-181.0]$ & -.009 & .993 \\
\hline \multicolumn{7}{|c|}{ Pressione arteriosa diastolica } \\
\hline Baseline & $71.5(18.1)$ & $70.0[34.0-155.0]$ & $69.2(12.5)$ & 70.0 [39.0-104.0] & -.615 & .538 \\
\hline Post-test & $69.2(12.5)$ & 70.0 [39.0-104.0] & $69.6(14.1)$ & 70.0 [40.0-111.0] & -.077 & .939 \\
\hline Wilcoxon Test & $Z=-.301$ & $p=.763$ & $Z=-.030$ & $p=.976$ & & \\
\hline \multicolumn{7}{|l|}{ Glicemia } \\
\hline Baseline & $115(37.2)$ & $107.0[69.0-243.0]$ & $126.8(40.7)$ & $123.0[63.0-251.0]$ & -1.683 & .092 \\
\hline Post-test & $118(39.1)$ & $111.0[62.0-232.0]$ & $121.1(33.6)$ & $112.0[76.0-214.0]$ & -.635 & .526 \\
\hline Wilcoxon Test & $Z=-1.262$ & $p=.207$ & $Z=-2.102$ & $p=.036$ & & \\
\hline \multicolumn{7}{|c|}{ Saturazione ossigeno } \\
\hline Baseline & $97.9(.82)$ & $98.0[95.0-99.0]$ & $97.7(.8)$ & $98.0[95.0-99.0]$ & -1.105 & \\
\hline Post-test & $97.8(.86)$ & 98.0 [95.0-100.0] & $98.9(.64)$ & 99.0 [97.0-100.0] & -6.517 & .269 \\
\hline Wilcoxon Test & $Z=-1.134$ & $p=.257$ & $Z=-6.303$ & $p=.000$ & & .000 \\
\hline \multicolumn{7}{|l|}{ VASM } \\
\hline Wilcoxon Test & $Z=-1.000$ & $p=.317$ & $Z=-6.735$ & $p=.000$ & & \\
\hline
\end{tabular}

VASP = visual analogue scale for pain VASM = visual analogue scale for mood; VASI = visual analogue scale for itching. Test a due code a livello di significatività a $p<0.05$.

ti nei livelli di saturazione nei neonati prematuri dopo l'ascolto della musica $(20,21)$. Un secondo risultato di questo trial è la riduzione statisticamente significativa del livello di dolore all'interno del gruppo che ascoltava la musica. Questo risultato è in linea con la revisione sistematica della Cochrane già menzionata, la quale suggerisce la possibilità di riduzione del livello del dolore correlato all'ascolto della musica. Esistono diversi meccanismi fisiologici attivati dalla musica nella riduzione del dolore. La "Teoria del Cancello" (22) sostiene che la musica agisca sulle fibre sensoriali di tipo II (gruppo $A \beta$ ), le quali inducono i neuroni inibitori a bloccare la trasmissione degli impulsi dolorosi al cervello; in questo modo, il cancello si ritiene chiuso e i pazienti avver- tono una riduzione della percezione dolorosa (23). Un'altra spiegazione è l'attivazione dei processi mentali legati alla concentrazione, la quale potrebbe modificare la trasmissione degli impulsi dolorosi a livello del midollo spinale, procurando stimoli antagonisti agli impulsi nervosi periferici. Ciò può mascherare anche gli stimoli ambientali ospedalieri, che possono innalzare o ridurre la percezione del dolore, aumentare i livelli endorfinici e incentivare dei processi di immaginazione, distraendo il paziente dai pensieri legati al dolore (24). Un altro risultato statisticamente significativo è stato il miglioramento dello stato dell'umore nel gruppo che ascoltava la musica. Anche in questo caso, i nostri dati concordano con la revisione sistematica della Cochrane prima 


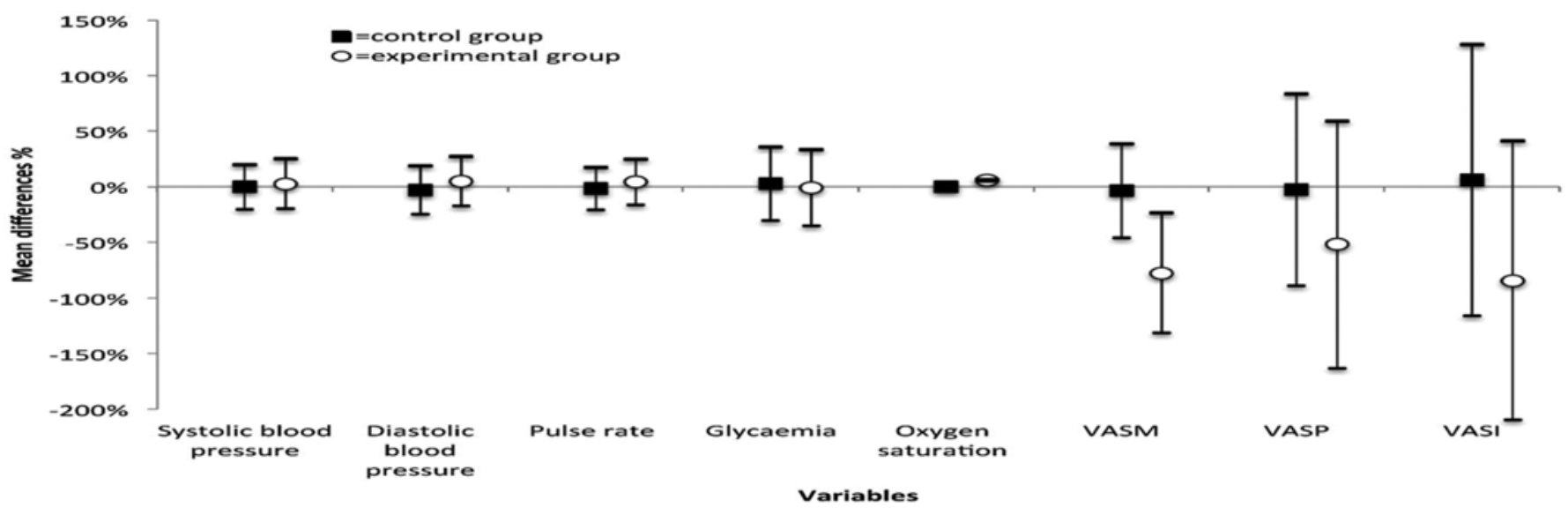

Fig. 3 - Differenze medie in percentuale tra i gruppi.

menzionata, la quale presenta dati a favore dell'intervento musicale sul miglioramento dello stato d'animo nei pazienti. La musica può migliorare l'umore per via psicosomatica e per via psiconeuroendocrinoimmunologica. Inducendo un rilassamento fisico (25), la musica influenza i livelli della morfina6-glucuronide e dell'interleukina-6 (26), innalza i livelli della serotonina e dell'endorfina, riduce i livelli delle catecolamine (27) e aumenta l'attività delle onde cerebrali $\alpha$. Inoltre, induce uno stato di rilassamento psicologico grazie all'attivazione di processi legati all'immaginazione e alla sfera cognitiva ed emotiva (28), migliorando l'umore (29-32). Infine, un risultato statisticamente significativo è dato dalla riduzione del livello di prurito nei pazienti del gruppo sperimentale. Nello studio di Terrill (33), la musica rappresenta uno dei trattamenti complementari contro i problemi connessi al prurito. Nell'ambito dell'assistenza infermieristica, esistono differenze rilevanti tra la musica registrata e quella suonata dal vivo. Durante un intervento musicale dal vivo, il rapporto tra musicista e paziente è dinamico, continuativo e non verbale. La musica diventa il mezzo comunicativo della sfera emotiva tra musicista e paziente. Durante l'ascolto musicale, il paziente osserva i movimenti del corpo e i gesti del musicista, il quale, a sua volta, modifica e modula l'intervento secondo la comunicazione verbale e non verbale del paziente. Questo genere di empatia non può sussistere durante l'ascolto della musica registrata da parte del paziente.

\section{Limiti}

Questo studio presenta diversi limiti. Lo studio era monocentrico, con l'arruolamento dei pazienti effettuato solo in una singola unità operativa emodialitica. Questa condizione di setting non ha permesso un confronto dei dati con eventuali dati di altre unità emodialitiche o di altri ospedali, riducendone la generalizzazione. Il campione era poco numeroso, il che non ha permesso di usare una statistica para- metrica. Lo studio non presenta un follow-up per studiare gli effetti nel tempo. L'intervento musicale è stato eseguito solo da un'infermiera-musicista, dunque non è stato possibile determinare se il miglioramento degli outcome sia legato anche al tipo relazione che la musicista ha saputo instaurare con i pazienti.

\section{Implicazioni per la pratica}

In un reparto emodialitico che abbia una visione olistica, un infermiere olistico dotato di preparazione musicale e che sia capace di suonare uno strumento musicale dal vivo potrebbe essere parte integrante nell'assistenza infermieristica quotidiana. L'intervento musicale potrebbe essere formalizzato all'interno dell'organizzazione e nei protocolli operativi.

\section{Conclusioni}

Questo articolo presenta uno studio sperimentale pilota basato sulle evidenze scientifiche riguardo all'introduzione della musica dal vivo, come intervento olistico, non invasivo e non farmacologico, all'interno di un'unità operativa di emodialisi. I risultati mostrano che l'uso della musica potrebbe favorire una riduzione del dolore, ridurre l'intensità del prurito, aumentare la saturazione arteriosa e migliorare la condizione dell'umore nei pazienti sottoposti a emodialisi e non presenta effetti indesiderati o collaterali.

\section{Note di pubblicazione}

Questo articolo è basato sul primo studio di Francesco Burrai, Valentina Micheluzzi, Maria Pia Zito, Pietro Giurdanella e Davide Sisti. Effects Of Live Saxophone Music On Physiological Parameters, Pain, Mood And Itching Levels In Patients Undergoing Haemodialysis. Journal of Renal Care, Volume 40, Issue 4, pages 249-256, December 2014. DOI: 10.1111/jorc.12078.

L'autorizzazione alla traduzione e alla pubblicazione è 
stata ottenuta dal John Wiley and Sons and Copyright Clearance Center, con numero di licenza 3696400698249, in data 26 Agosto 2015.

Ha collaborato alla traduzione dell'articolo Roberta Mereu, Infermiera,UO Nefrologia Dialisi e Trapianto-La Manna, AOU di Bologna. Membro dell'Italian EDTNA/ERCA Research Center.

\section{Disclosures}

Financial support: No financial support was received for this submission.

Conflict of interest: The author has no conflict of interest.

\section{Bibliografia}

1. Ostermann T, Boyde T, Linden U. Music therapy in the treatment of cancer patients: a systematic review. BMC Complementary and Alternative Medicine 2012;12(1):327.

2. Richards T, Johnson J, Sparks A, et al. The effect of music therapy on patients' perception and manifestation of pain, anxiety, and patient satisfaction. Medsurg Nurs 2007;16(1):7-14.

3. Dochterman JM, Bulechek GM. Nursing Interventions Classification (NIC). St Louis: Mosby 2004

4. Joanna Briggs Institute. The Joanna Briggs Institute Best Practice Information Sheet: music as an intervention in hospitals. Nurs Health Sci 2011;13(1):99-102.

5. McCaffery M. Nursing approaches to nonpharmacological pain control. Int J Nurs Stud 1990;27(1):1-5.

6. Cassileth BR, Vickers AJ, Magill LA. Music therapy for mood disturbance during hospitalization for autologous stem cell transplantation: a randomized controlled trial. Cancer 2003; 98(12):2723-9.

7. Hilliard RE. The effects of music therapy on the quality and length of life of people diagnosed with terminal cancer. J Music Ther 2003;40(2):113-37.

8. Gallagher LM, Lagman R, Walsh D. The clinical effects of music therapy in palliative medicine. Support Care Cancer 2006;14(8):859-66.

9. Caminha LB, da Silva MJ, Leão ER. [The influence of musical rhythms on the perception of subjective states of adult patients on dialysis]. Rev Esc Enferm USP 2009;43(4):923-9.

10. Schuster I. The effect of music listening on blood pressure fluctuations in adult hemodialysis patients. Journal of Music Therapy 1985;22(3):146-53.

11. Martinez J. Is music therapy? Nephrol Nurs J 2009;36(3):329-30.

12. Lin YJ, Lu KC, Chen CM, et al. The effects of music as therapy on the overall well-being of elderly patients on maintenance hemodialysis. Biol Res Nurs 2012;14(3):277-85.

13. Cantekin I, Tan M. The influence of music therapy on perceived stressors and anxiety levels of hemodialysis patients. Ren Fail 2013;35(1):105-9.

14. Hedayati SS, Yalamanchili V, Finkelstein FO. A practical approach to the treatment of depression in patients with chronic kidney disease and end-stage renal disease. Kidney Int 2012; 81(3):247-55.

15. Price DD, Bush FM, Long $S$, et al. A comparison of pain measurement characteristics of mechanical visual analogue and simple numerical rating scales. Pain 1994;56:217-26.

16. Good M, Chin CC. The effects of Western music on postoperative pain in Taiwan. Kaohsiung J Med Sci 1998;14(2):94-103.

17. Chang MY, Wang SY, Chen CH. Effects of massage on pain and anxiety during labour: a randomized controlled trial in Taiwan. J Adv Nurs 2002;38(1):68-73.

18. Chan MF, Chan EA, Mok E, et al. Effect of music on depression levels and physiological responses in community-based older adults. Int J Ment Health Nurs 2009;18(4):285-94.

19. Bradt J, Dileo C, Grocke D, et al. Music interventions for improving psychological and physical outcomes in cancer patients. Cochrane Database Syst Rev 2011;(8):CD006911.

20. Cignacco E, Hamers JP, Stoffel L, et al. The efficacy of nonpharmacological interventions in the management of procedural pain in preterm and term neonates. A systematic literature review. Eur J Pain 2007;11(2):139-52.

21. Hartling L, Shaik MS, Tjosvold L, et al. Music for medical indications in the neonatal period: a systematic review of randomised controlled trials. Arch Dis Child Fetal Neonatal Ed 2009;94(5):F349-54.

22. Melzack R, Katz J. The Gate Control Theory: Reaching for the Brain. In: Craig KD, Hadjistavropoulos T. Pain: psychological perspectives. Mahwah, N.J: Lawrence Erlbaum Associates, Publishers; 2004

23. Crowe BJ. Music and Soulmaking: Toward a New Theory of Music Therapy. (Lanham, MD:The Scarecrow Press 2004),119.

24. Boso M, Politi P, Barale F, et al. Neurophysiology and neurobiology of the musical experience. Funct Neurol 2006;21(4):187-91.

25. Igawa-Silva $\mathrm{W}, \mathrm{Wu} S$, Harrigan R. Music and cancer pain management. Hawaii Med J 2007;66(11):292-5.

26. Stefano GB, Zhu W, Cadet P. Music alters constitutively expressed opiate and cytokine processes in listeners. Med Sci Monit 2004;10(6):MS18-27.

27. Mok E, Wong KY. Effects of music on patient anxiety. AORN J 2003;77(2):396-7,401-6,409-10.

28. Kemper KJ, Danhauer SC. Music as therapy. South Med J 2005; 98(3):282-8.

29. Lai HL, Chen CJ, Peng TC, et al. Randomized controlled trial of music during kangaroo care on maternal state anxiety and preterm infants' responses. Int J Nurs Stud 2006;43(2):139-46.

30. Bailey LM. The effects of live music versus tape-recorded music on hospitalized cancer patients. Music Therapy 1983;3(1): 1728. 13.14

31. Burns SJ, Harbuz MS, Hucklebrideg F, et al. A pilot study into the therapeutic effects of music therapy at a selfhelp cancer center. Altern Ther Health Med 2001;7(1):48-56.

32. Barrera ME, Rykov MH, Doyle SL. The effects of interactive music therapy on hospitalized children with cancer: a pilot study. Psychooncology 2002;11(5):379-88.

33. Terrill B. Renal Nursing: A Practical Approach. Melbourne: Ausmed Publications 2002 Corretto! 